\title{
Magnetic Alignment of Iron(III) Oxide Decorated CNT Polymer Composites
}

\author{
E. L. Camponeschi, J. W. Walker, H. Garmestani, and R. Tannenbaum* \\ * Department of Materials Science and Engineering, Georgia Institute of Technology School of \\ Engineering, Atlanta, GA 30332
}

\section{Introduction}

In this work we have developed a new way of magnetically aligning the carbon nanotubes to allow complete alignment of the composite sample as the sample was being processed, thus avoiding the pitfalls of the other methods. Since carbon nanotubes are not magnetic, and can not align on their own in a magnetic field, a cooperative influence is necessary. This can take the form of other aligning fibrous networks (such as a polymer matrix), ${ }^{1,2}$ or tethering to them a magnetic material. ${ }^{1}$ Thus, the focus of this work is to tether iron oxide nanoparticles to the carbon nanotubes in order to facilitate their alignment while embedded in a polymer matrix.

\section{Experimental Procedure}

The composites were made with Araldite GY 6008 (Huntsman Advanced Materials Americas Inc.), the resin is a Bisphenol A Diglycidyl ether, and the hardener used was ANCAMINE AEP curing agent (Air products), and is an aminoethyl piperazine, 1-(2-, (AEP).

The iron oxide particles were manufactured utilizing a modified sol-gel processing method, which creates nano iron oxide particles at room temperature. The dried iron oxide batches were then added to the carbon nanotubes. Solutions were made with $0,0.5$, or $1.0 \mathrm{wt} \%$ carbon nanotubes. The hardener was added to the resin/CNT/iron oxide mixture, and the mixture was poured into Teflon molds and placed in a magnetic field. The samples were heated to $100^{\circ} \mathrm{C}$ for several hours and allowed to harden in 0.0 (no magnetic field), 0.4, and 0.8 Tesla magnetic fields, Table 1.

\section{Results}

The initial goal of using a magnetic field on carbon nanotube composites was to promote the directional alignment of the carbon nanotubes, which would improve the Young's modulus of the composite. In order to analyze this potential improvement, we used Differential Scanning Calorimetry to measure the changes in glass transition temperature; compression testing to measure the compression strength change; and Raman spectroscopy to determine carbon nanotube alignment.

\section{Conclusions and Recommendations}

In this work, we have analyzed the effect of a magnetic field on carbon nanotube alignment in epoxy-based composites. This work has illustrated that the magnetic field had a positive impact on the mechanical properties of the epoxy used (Figure 1). The alignment of carbon nanotubes in these samples was difficult to determine accurately and was not uniform for all samples (Figure 1). The reason for the non-uniformity is believed to be due to the concentration of iron oxide tethered to the surface of the carbon nanotubes, the magnetic field strength, and the variance in viscosity during curing. At 0.4 Tesla a lower concentration of iron oxide was needed to induce alignment, whereas at 0.8 Tesla a higher concentration of iron oxide was needed, results which are counterintuitive.

However, taking into consideration the results of the alignment experiments coupled with the characteristics of the processing methods and the changes of the viscosity in the samples, the reasoning behind the inconsistent results becomes clearer. The viscosity of the epoxy was strongly dependent on temperature, and the ability to quickly place the CNT-iron oxide-epoxy mixture into the mold and in the magnetic field. The work presented here points to the applicability of this in situ processing method for the creation of a magnetically-induced aligned epoxy composite. However, 
the processing method needs to be further studied and most likely modified, in order to create uniformly aligned samples.

References

[1] Camponeschi, E., et al. Carbon. 45(10) (2007) 2037.

[2] Camponeschi, E. Dispersion and Alignment of Carbon Nanotubes in Polymer Based Composites. PhD Thesis. Georgia Institute of Technology. 2007.

[3] This work was supported by Institute of Paper Science and Technology at Georgia Tech through the Otto Kress Scholarship.

TABLE 1. Sample Parameters

\begin{tabular}{|c|c|c|c|c|l|}
\hline Sample \# & $\mathbf{T}_{\mathbf{g}}\left({ }^{\mathbf{0}} \mathbf{C}\right)$ & $\mathbf{C N T}(\mathbf{w t} \%)$ & $\begin{array}{c}\mathbf{F e}_{\mathbf{2}} \mathbf{O}_{\mathbf{3}} \\
\text { batches }\end{array}$ & $\begin{array}{c}\text { Magnetic } \\
\text { Field (Tesla) }\end{array}$ & Raman Analysis \\
\hline 1 & 54.9 & 0.0 & 0 & 0 & \\
\hline 2 & 41.6 & 0.5 & $1 \mathrm{x}$ & 0 & \\
\hline 3 & 45.5 & 1.0 & $1 \mathrm{x}$ & 0 & \\
\hline 4 & 68.7 & 0.0 & 0 & 0.4 & \\
\hline 5 & 86.0 & 0.5 & $1 \mathrm{x}$ & 0.4 & Alignment \\
\hline 6 & 60.8 & 0.5 & $2 \mathrm{x}$ & 0.4 & \\
\hline 7 & 50.7 & 0.5 & $2 \mathrm{x}$ & 0.4 & \\
\hline 8 & 46.2 & 1.0 & $1 \mathrm{x}$ & 0.4 & Alignment \\
\hline 9 & 75.1 & 1.0 & $2 \mathrm{x}$ & 0.4 & \\
\hline 10 & 50.6 & 1.0 & $2 \mathrm{x}$ & 0.4 & \\
\hline 11 & 103.4 & 0.0 & 0 & 0.8 & \\
\hline 12 & 49.8 & 0.5 & $1 \mathrm{x}$ & 0.8 & Alignment \\
\hline 13 & 58.9 & 0.5 & $2 \mathrm{x}$ & 0.8 & \\
\hline 14 & 45.9 & 1.0 & $1 \mathrm{x}$ & 0.8 & \\
\hline 15 & 55.3 & 1.0 & $2 \mathrm{x}$ & 0.8 & \\
\hline 16 & 87.9 & 1.0 & $2 \mathrm{x}$ & 0.8 & Alignment \\
\hline 17 & 44.6 & 0.0 & 0 & 0 & \\
\hline
\end{tabular}
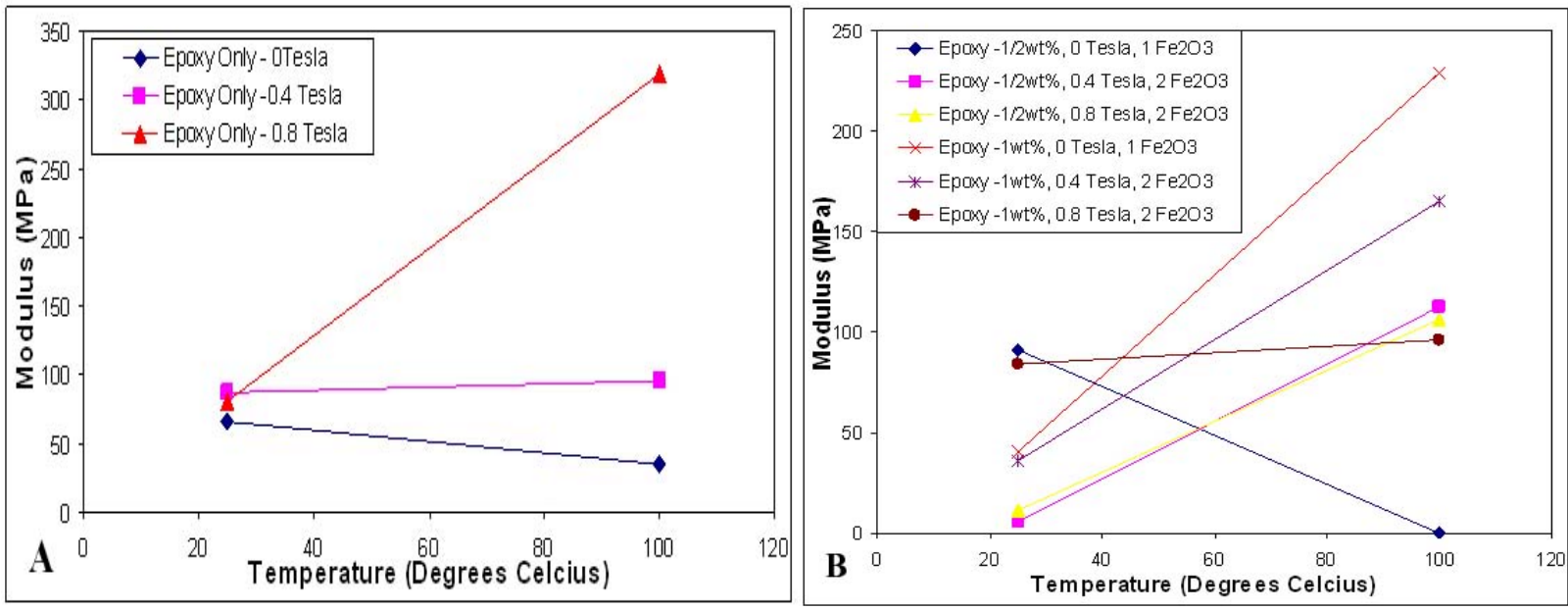

FIG. 1. Elastic Modulus of the samples at room temperature $\left(25^{\circ} \mathrm{C}\right)$ and above $\mathrm{T}_{\mathrm{g}}\left(100^{\circ} \mathrm{C}\right)$, (a) epoxy only data, (b) composite data. 